\title{
Study on Cloud Computing Data Center Infrastructure Best Practices
}

\author{
Shuguang $\mathrm{Qi}^{1}$, Bin $\mathrm{Yu}^{2}$ \\ China Academy of Telecommunication Research of MIIT, China Telecommunication Technology \\ Labs, Beijing, China
}

\begin{abstract}
Cloud computing data center infrastructure new technologies and best practices are analyzed in this paper. First of all, modular cloud computing data center which is divided into building type and container type is described. In addition, principles and energy efficiencies of new cooling methods in practical application in cloud computing data center are analyzed. Finally, HVDC (Higher Voltage Direct Current) power feeding system structure, voltage ranges and energy efficiency are studied.

Keywords

Cloud Computing Modular Data Center, Energy Efficiency, Outdoor Cooling, Heat Exchanging and Evaporative Cooling, Spot Cooling, HVDC
\end{abstract}

\section{Introduction}

Data Center technology is improving every day. New servers allow for higher densities than ever before, putting great strains on infrastructures such as legacy cooling and power products. In response, corporations need to intelligently allocate resources to get the most from their data center infrastructure. Optimizing current environment with the right cooling and power infrastructure will help to reclaim precious capacity and may allow to delay or avoid altogether the time and expense required to build a new data center. Therefore in this paper, new technologies in cloud computing data center infrastructure and corresponding energy efficiencies are analyzed.

\section{Cloud computing modular data center}

The infrastructure in the cloud computing modular data center space should consist of the common facilities space and modularized facilities space by module. The common facilities space is not affected by the module, e.g., main power receiving and transformation facilities, whereas the modularized facilities should include UPS system (or HVDC system) and cooling system. Independent capacity and power density will be supported by configuring the individual facilities independently for each module of the cloud computing modular data center. The facilities by in every module including low-voltage power distribution unit, AC UPS or HVDC, and cabling should be designed according to the requirements of the IT equipment extension. Therefore, modular design has very good flexibility comparing with common data center infrastructure configuration. The infrastructure composition by module will be differentiated by module according to the availability and reliability requirement.

There are two kinds of cloud computing modular data center in the real application. One is building type and the other is container-type.

2.1 building-type cloud computing modular data center

The building-type cloud computing modular data center constructs individual server rooms in the center on a scale large squire building. Different modules were put in the one big data center, every modules has it's own power feeding and cooling unit to ICT equipment. Therefore it is not necessary to cool all big data center. Compared with the container center, there are limitations in terms of the place and construction period. However, that it is more flexibility, high-density, high-integrity and suitable for the cloud computing data center.

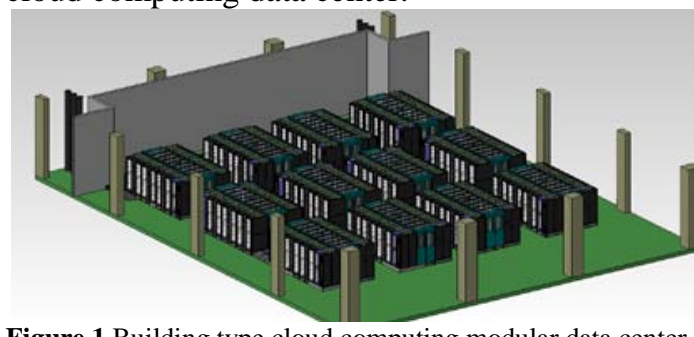

Figure.1 Building type cloud computing modular data center 2.2 Container-type cloud computing modular data center

The container-type cloud computing data center integrates various IT infrastructures in a cargo container. When this type of center is introduced, high-density servers can guarantee higher energy efficiency than the existing center by overcoming the limitation of the place and infrastructure. It is also allows flexible extension, since the container park-type, large-size center can be built by piling up container centers.

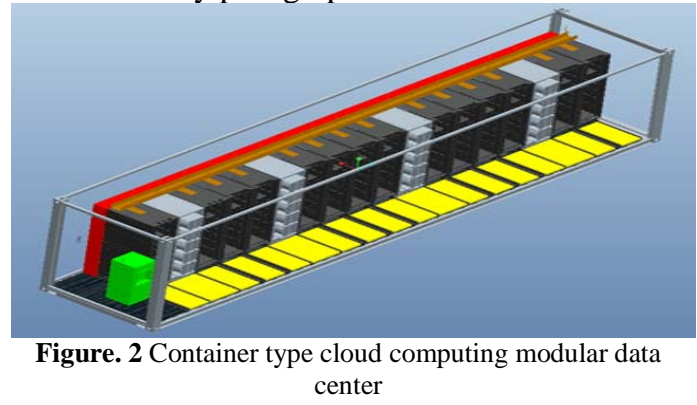




\section{Analysis on cooling system}

There are many methodologies for cooling data centers with high density ICT devices, including outdoor cooling, spot cooling, evaporative cooling, heat exchanging cooling, and hybrid cooling etc. Several typical cooling methods are introduced and energy efficiencies are analyzed in this chapter.

\subsection{Outdoor cooling}

Figure 3 shows the configuration of the outdoor air cooling system. With outdoor cooling, in addition to floor-supply air conditioners supplying cooled air to the room as with conventional air conditioning, the air conditioning system incorporates exhaust fans discharging air from the room to the outside, and outdoor air ducting introducing outdoor air to the air conditioners.

With this method, as with conventional air conditioning, air cooled with multiple floor supply air conditioners installed in the room is supplied to the room for cooling. During the intermediate seasons, and in winter when outdoor air temperature is low, outdoor air is introduced directly to the air conditioner to reduce the amount of cooling required by the cooling coils, and water chilling unit energy consumption for generating chilled water is greatly reduced.

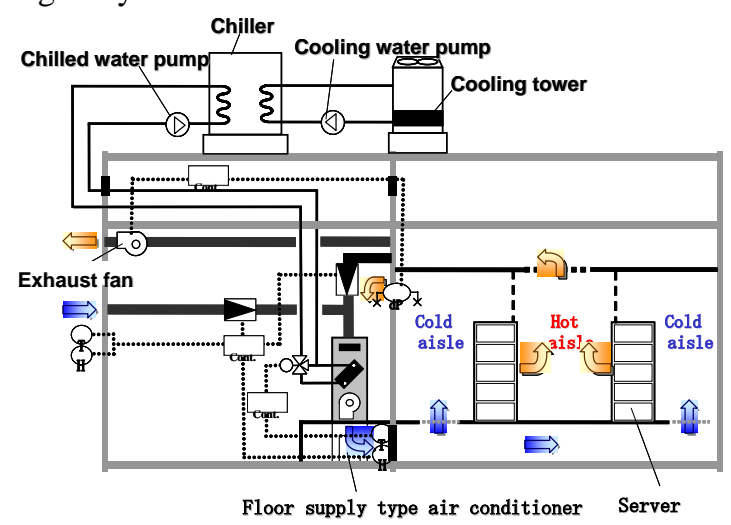

Figure 3 Outdoor cooling with conventional air conditioning system

3.2 Heat exchanging and evaporative cooling Figure 4 shows the configuration of the heat exchanging and evaporative cooling system. As with a conventional system, it employs floor supply air conditioners to supply cold air to the room. Furthermore, the equipment is made up of an evaporative cooling unit comprising an evaporative cooler to cool return air passed from the room to the air conditioner with outdoor cold air, and an indirect sensible heat exchanger, an outdoor air fan passing outdoor air through the evaporative cooling unit, and a circulation fan passing air through the evaporative cooling unit. A large number of server devices are installed in server racks on the raised floor in the room, arranged in a regular pattern alternately facing the cold aisle (towards which the server device air inlets are directed) and the hot aisle (towards which the exhausts are directed). The high-temperature interior air discharged from the hot aisle into the space at the top of the room is drawn in from the top of the floor supply air conditioners, dehumidified and cooled to specifications using the cooling coils in the air conditioner, and supplied to the under-floor chamber. Part of the air circulated in the air conditioner from the hot aisle is introduced to the evaporative cooling unit. Similarly, outdoor air is introduced into the evaporative cooling unit, and outdoor air humidified in the evaporative cooler, and the resulting cooled air and return air exchange heat indirectly so that humidity remains unchanged while the temperature is reduced. Thus, the interior return air cooled with the latent heat of evaporation of water is mixed with interior return air circulated directly in the air conditioner, dehumidified and cooled to the specified temperature using chilled water in the cooling coils in the air conditioner, and supplied to the under-floor chamber.

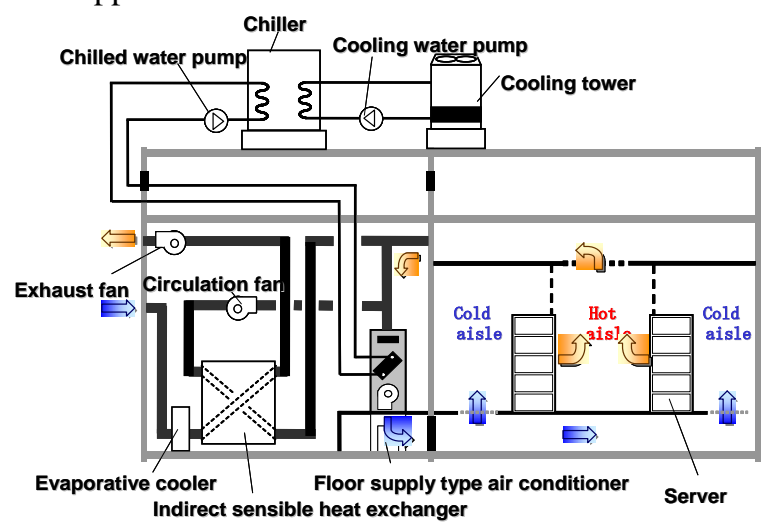

Figure4. Heat exchanging and evaporative cooling with

\subsection{Spot cooling}

Figure 5 shows the configuration of the spot cooling system used for natural circulation of the refrigerant. The combined spot cooling method and typical air conditioning method, and the spot cooling method alone, are possible for cooling. A large number of server racks incorporating server devices are installed on the free access floor in the room, and are arranged alternately facing the cold aisle and the hot aisle.

With the spot cooling method, a spot cooling unit cooling the air in the hot aisle and circulating it in the cold aisle, and a water-cooled condenser using chilled water to condense refrigerant gas evaporated with the spot cooling units, were installed. Suspended spot cooling units employing natural circulation of refrigerant were installed in the space between the server rack and the ceiling. These units draw in the high-temperature return air discharged from the hot aisle into the space return air in the room to the specified temperature, and supply it 
to the cold aisle.

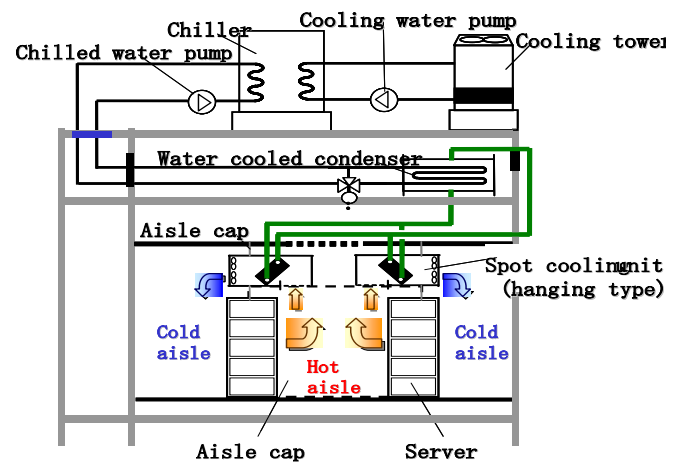

Figure5. Spot cooling with conventional air conditioning system

With this method, the spot cooling units cool the air locally, and are thus effective in preventing hot spots. Furthermore, by handling locally the entire amount of heat generated in the room, the blowers supplying large volumes of air to the entire room, as with the typical air conditioning method, are substituted by a small fan for localized air circulation, and a large reduction in heat transport power is possible.

\subsection{Energy efficiency analysis on different cooling methods}

Test results showed chilling system power was greatly reduced by effective use of outdoor air with outdoor air cooling in temperate and cold regions, with a reduction in chilling system power of approximately 57\% in Tokyo, 78\% in Moscow, and 98\% in London. However, with nearly double the transport power over conventional air conditioning due to increased air conditioner resistance and the installation of exhaust fans, the reduction in total conditioning power consumption was $28 \%$ in Moscow, and $39 \%$ in London.

Chilling system power was also reduced with the heat exchanging and evaporative cooling system by effective use of outdoor air, and while energy conservation was slightly inferior to that with outdoor air cooling, since only indirect heat exchange is used, the reduction in chilling system power consumption was 35\% a cold location like Moscow and 23\% in London. However, since the transport power of the ventilation fan in evaporative cooling units is greater than with conventional air conditioning, the reduction in total air conditioning power consumption was 3\% in London, and $8 \%$ in Moscow.

With spot cooling, the use of natural circulation of refrigerant, and localized handling of heat with a spot cooling unit, reduced the transport power necessary for heat transport dramatically in comparison with conventional air conditioning, and power consumption was able to be reduced to a stable level without being affected by outdoor air conditions. With this method, considerable benefits were obtained through reduction of transport power, even where outdoor air is not effective (e.g. temperate regions). Reductions of $22 \%$ were obtained in tropical Singapore, 21\% in London, and 22\% in Moscow.

Therefore, cooling methods should be selected according to different climatic in different regions, so as to higher cooling efficiency can be achieved.

\section{Analysis on HVDC power feeding system}

4.1 HVDC power feeding system structure Up to $400 \mathrm{~V}$ Direct current system, as it's output voltage is much higher than $-48 \mathrm{~V}$ voltage system, is called HVDC system. It's structure is shown in Figure6 ${ }^{[3]}$. System consists of AC distribution, rectifiers, DC distribution, and insulation monitors etc.

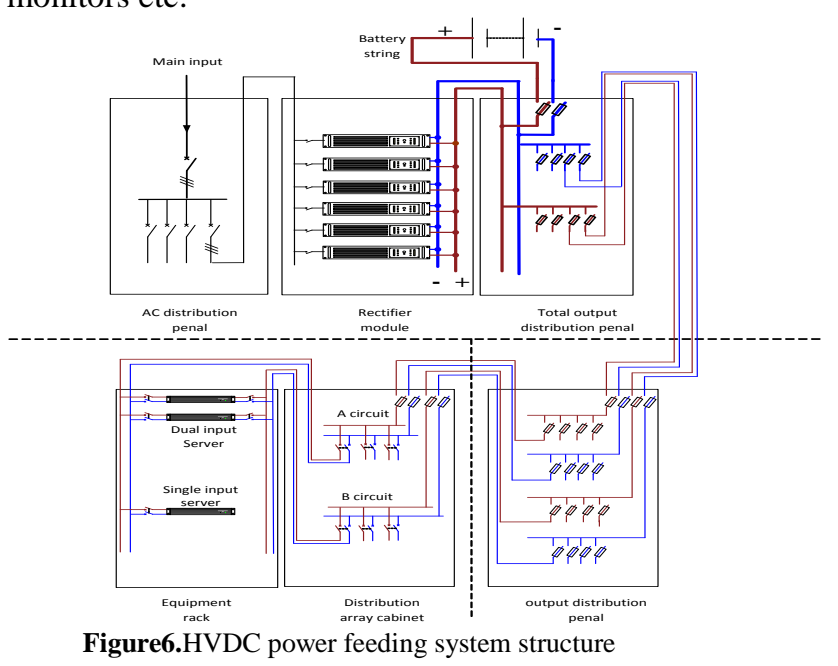

4.2 HVDC power feeding system interface Interface $\mathrm{P}$ in Figure 7 is a physical point of power feeding system and ICT equipment connection.

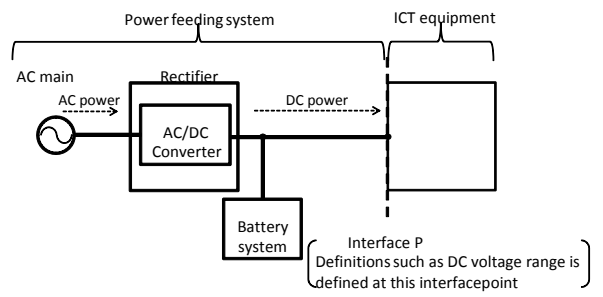

Figure7. Illustration of power feeding system and ICT equipment

4.3 HVDC power feeding system output voltage ranges at interface $P$

Voltage ranges according to ITU-T L.1200 is divided into target solution voltage range and transitional solution voltage range.

(1) Target solution 260V-400V

(2) Transitional solution

192V-288V

(3) Abnormal DC voltage range for target solution

- $0 \mathrm{~V}<\mathrm{U}<260 \mathrm{~V}$. 
- $400 \mathrm{~V}<\mathrm{U}<410 \mathrm{~V}$.

After the restoration of the supply from the abnormal DC voltage range to the normal DC voltage range, the ICT equipment shall not incur any damage and will be able to automatically resume operation.

\subsection{Energy efficiency analysis on HVDC} power feeding system

In traditional AC UPS power system, 1+1 is very common configuration, the highest load rate of AC UPS is $50 \%$. Based on our study on 36 different mode UPS systems energy efficiency, test result is shown in the Figure 8. $\mathrm{EE}$ in the Figure 8 and Figure 9 is the Abbreviation of energy efficiency.

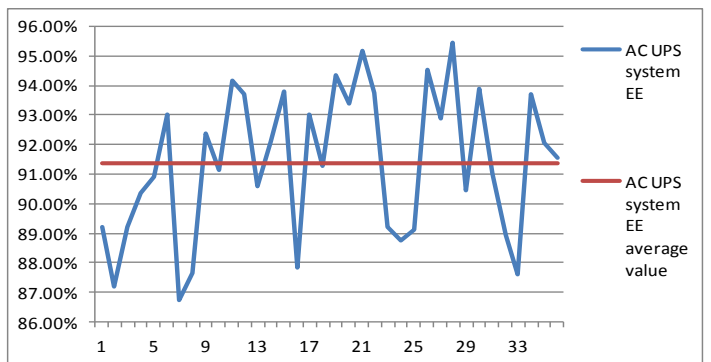

Figure.8 Energy efficiency of AC UPS in 50\% load condition

From the figure it can be seen that in 1+1 configuration, AC UPS system efficiency average value red line in figure 8 is about $91.4 \%$.

In AC UPS system, the input voltage is converted to DC voltage by AC/DC converting circuit. The voltage is converted back to $\mathrm{AC}$ voltage by DC/AC inverter. As well as for HVDC system, the input voltage is first converted to DC by one phase PFC circuit or three phase PFC circuit. It is almost or much higher than $400 \mathrm{~V}$. Therefore, there must be another voltage conversion DC/DC circuit to decrease output voltage to system working voltage $380 \mathrm{~V}$ (for target solution) or $276 \mathrm{~V}$ (for transitional solution). So it can be concluded that there are two voltage conversions in both in AC UPS and HVDC system.

As for HVDC system, as it is modular design and directly connected to the battery string, it is not necessary to configure as $1+1$ system. Therefore, HVDC system efficiency test in 100\% load condition, the test result is shown in Figure 9. From the Figure it can be seen that $336 \mathrm{~V}$ HVDC system increases system efficiency to 95.4\%, and 240V HVDC system increases system efficiency to $93.5 \%$. It is much higher than 1+1 AC UPS system energy efficiency. Furthermore, as for AC UPS system, When Gird is off, the battery string is discharged through the DC/AC inverter. While for HVDC system, as battery string is directly connected to the load (ICT equipments), the reliability of the power feeding system highly increased comparing with the AC UPS system.

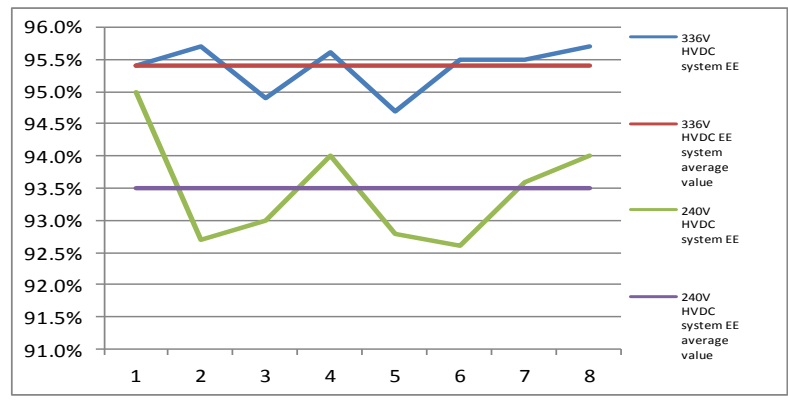

Figure.7 Energy efficiency of HVDC system in 100\% load condition

\section{Conclusion}

A data center is a facility used to house computers, telecommunications, and storage systems. Most data centers have redundant power systems and data communications connections, as well as proper cooling methods to protect the equipment and the clients' data. By developing new technologies in data center infrastructures, energy efficiency can be highly increased and reduces the cost of energy consumption and operation. Moreover, not only further study on energy efficiency of infrastructure, but also energy efficiency of whole data center evaluation method will be studied to increase whole cloud computing data center energy efficiency in the future.

\section{References}

[1] ITU-T L.1300 "Best practices for green data centres"

[2] ITU-T L.1200 "Direct current power feeding interface up to $400 \mathrm{~V}$ at the input to telecommunication and ICT equipment”

[3]CCSA-YD/T 2378-2011“240V Direct Current Power Supply System for telecommunications” [4] ShuguangQi,FupingHou, HuiJing, Study and Application on High Voltage DC Power Feeding System for Telecommunications in China, in Proc. of the 34th International Telecommunications Energy Conference (INTELEC 2012), 2012.

[5] Domagoj Talapko, Telecom Datacenter Power Infrastructure Availability Comparison of DC and AC UPS, in Proc. of the 34th International Telecommunications Energy Conference (INTELEC 2012), 2012.

[6] ShuguangQi, Reliability and Energy Saving Characteristics Analysis on New Power Feeding Mode in Telecommunications, Modern science \& technologies of telecommunications[J], Vol. 42 No.10, Pages (6-11). 